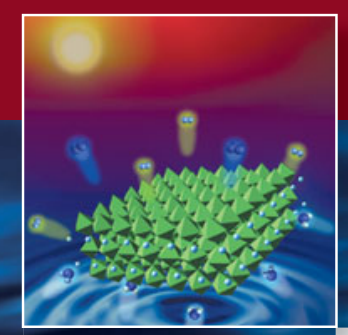

\title{
Recent developments in solar water-splitting photocatalysis
}

\author{
Frank E. Osterloh and Bruce A. Parkinson, Guest Editors
}

\begin{abstract}
Although photovoltaic cells have great potential for supplying carbon-free energy, they suffer from the lack of an efficient and cost-effective energy storage process that can supply energy for transportation and nighttime use. A direct way to convert solar energy into chemical fuels would solve this problem. Of several possible schemes, the photon-driven electrolysis of water to produce hydrogen and oxygen has been studied most. Photoelectrolysis of water can be achieved with either self-supported catalysts or with photoelectrochemical cells. This article will introduce the basic principles of solar water splitting and highlight recent developments with semiconductor light absorbers and co-catalysts. The role of combinatorial approaches in identifying new metal oxide visible light-absorbing semiconductors will be briefly described, and the potential of using nanomaterials for more efficient devices will be discussed. Separate articles in this special issue will focus on recent developments in watersplitting concepts.
\end{abstract}

\section{Driving force for cheap solar fuels}

The world's population is increasing and is expected to continue increasing until at least mid-century. All of these new inhabitants will require water, food, shelter, transportation, and consumer products, all of which need energy to produce and/or deliver. The population increase, along with the rapid growth of many developing economies, indicates that the demand for energy may more than double by mid-century. Even though they are a finite resource, fossil fuels would be capable of meeting this demand, albeit at an increasing cost as the easy-to-extract sources are depleted. However, the increasing concentration of $\mathrm{CO}_{2}$ in the atmosphere, as a result of the combustion of these carbon-based fuels, is predicted to result in unacceptable changes in the Earth's climate. In addition, pollution such as sulfur dioxide emissions and oil spills also point to the need for carbon-free alternative energy sources. Nuclear power and wind are both commercially viable carbon-free technologies that can contribute to the present and future energy mix. However, finding a viable way to tap solar energy, the most abundant and universally available source of renewable energy, is urgently needed. ${ }^{1}$ One problem with solar energy is that it is quite diffuse, requiring large areas of solar collectors to harvest significant amounts of power. Therefore, cost reductions in the production of thin-film solar cells are needed to produce solar electricity at a cost that can compete with conventional power sources if the costs of carbon emissions and other pollution are included. The other "Achilles' heel" of solar energy is that it is difficult to store as fuel for nighttime and transportation uses. Since fuels account for more than $70 \%$ of current energy consumption, there is an urgent need to convert solar energy directly to fuels, ideally liquid fuels that are easy to transport and store. To try and quickly develop systems to directly convert solar energy to fuels, the Department of Energy recently awarded a five-year $\$ 125$ million research grant to establish a "Solar Hub" to improve what are now only ideas or inefficient laboratory demonstrations into a viable solar fuels technology operating at $10 \%$ efficiency or higher.

\section{Solar-based water splitting methods}

Plants can convert and store solar energy as complex molecules, such as carbohydrates and other biomass; however, photosynthesis is not very efficient, and the stored energy in plants and the large amounts of arable land needed to grow them compete with other land uses such as food production. Storing solar energy by splitting water into hydrogen and oxygen has long been considered a promising idea; in fact, in 1874, Jules Verne, recognizing the finite supply of coal and the possibilities of hydrogen derived from water electrolysis, made the comment that "water will be the coal of the future" (Jules Verne, The Mysterious Island (available at http://www.literatureweb.net/verne/mysteriousisland, 1874). However, as of yet,

Frank E. Osterloh, University of California, Davis; osterloh@chem.ucdavis.edu

Bruce A. Parkinson, Department of Chemistry and the School of Energy Resources, University of Wyoming; bparkin1@uwyo.edu

DOI: $10.1557 / \mathrm{mrs} .2010 .5$ 
there are no economical methods for fulfilling this prediction, partially because of problems of efficiently storing and handling hydrogen. In addition to generating the required $\mathrm{H}_{2}$ from photocatalytic water splitting, researchers have devised and tested a number of thermochemical cycles that use high temperatures generated in focusing solar concentrators to split water. ${ }^{2,3}$ This process also has its own distinct materials needs and will not be reviewed herein.

The cost per watt of solar photovoltaic electricity has been steadily decreasing, and current technology can mate solar cells with conventional water electrolysis units to produce hydrogen. If cost were no object, one could generate hydrogen from sunlight and water at very high efficiencies, however, the highefficiency tandem solar cells and an electrolyzer operating at high faradaic efficiency would be extremely expensive. What is needed are semiconducting materials that can accomplish the absorption of light, the separation of the photo-generated charge carriers, and the efficient transfer of the carriers to an electrolyte solution to produce hydrogen and oxygen from water directly in one inexpensive device. The semiconducting materials must be highly stable, so as not to degrade in 20 or more years due to exposure to sunlight and electrolyte; they must have conduction and valance band energies such that the photo-generated electrons are sufficiently reducing to produce hydrogen, and the holes are sufficiently oxidizing to produce oxygen from water. In addition, they must be inexpensive. The problem is that no material, or combination of materials, that meets these requirements is known. In this issue, we will review some of the approaches that are being used to discover and optimize water splitting via photoelectrochemical or photocatalytic processes. Yang et al. describes bioinspired approaches to improve catalysts for water electrolysis. Zhang emphasizes the promise of inorganic nanostructured materials. Maeda and Domen describe (oxy)nitride materials as a possible way to increase visible light absorption of water splitting photocatalysts. Finally, Kudo will address the same problem using z-scheme photocatalyst systems, in which separate semiconductors are coupled electrochemically or electronically to improve water splitting efficiency.

\section{Photoelectrochemical water splitting}

Although oxygen evolution was observed as early as 1968 by illuminating an electrode made from the rutile form of $\mathrm{TiO}_{2}$ in solution, ${ }^{4}$ Fujishima and Honda first pointed out the application of this concept to water photoelectrolysis in a series of experiments that used an $n$-type rutile crystal ${ }^{5,6}$ as part of a photoelectrolysis cell, shown in Figure 1a. Here, the interaction of light with $\mathrm{TiO}_{2}$ produces electron-hole-pairs, of which the holes oxidize water at the $\mathrm{TiO}_{2}$ surface to form oxygen, and the electrons migrate to the $\mathrm{Pt}$ counter electrode to reduce water to form hydrogen. It should also be pointed out that the conduction band of rutile is not negative enough to reduce water, and so in the original publications, a " $\mathrm{pH}$ bias" was present where the oxygen-producing side of the cell was basic with respect to the hydrogen-producing electrolyte. While rutile is stable

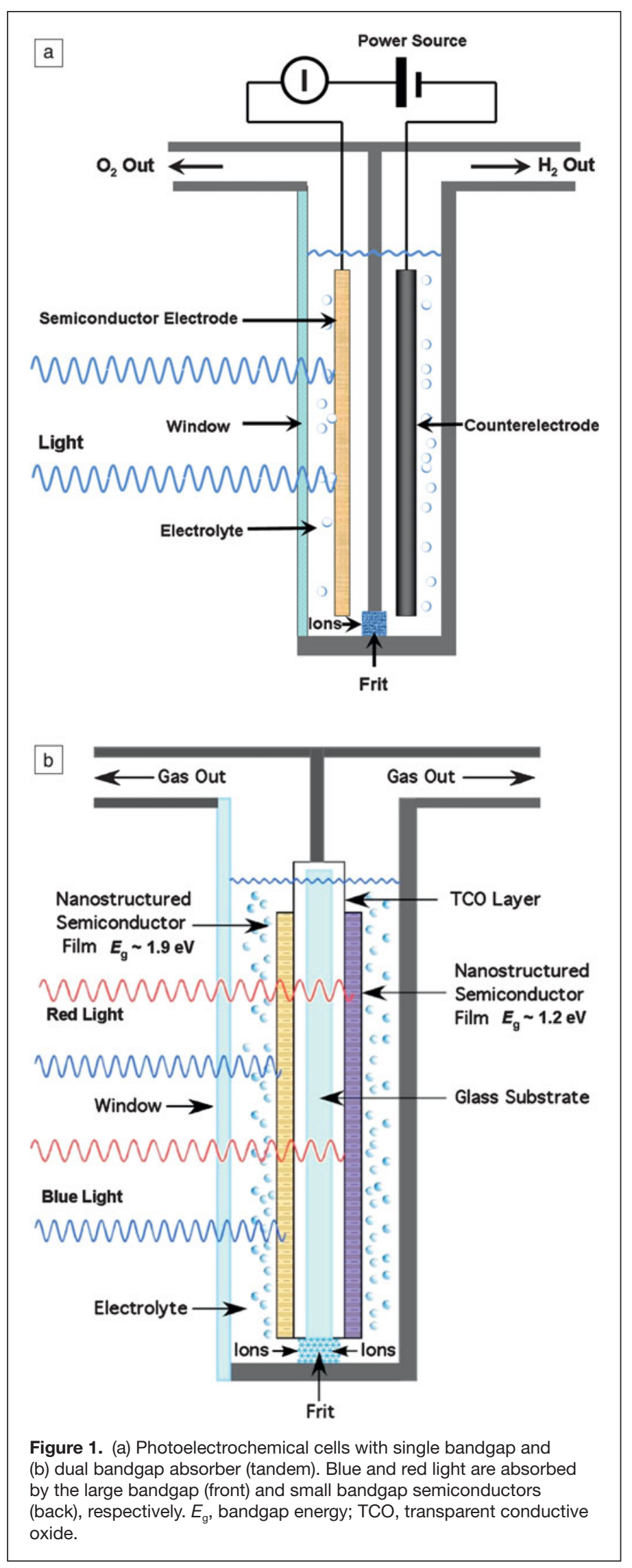


under illumination in aqueous electrolytes, its large bandgap $(3.0 \mathrm{eV})$ limits its utilization to the UV portion of the solar spectrum, and thus limits its ultimate efficiency. Later work, using heterojunctions of expensive and not completely stable III-V materials, such as a $\mathrm{GaInP}_{2}$ and a $n-p$-GaAs junction photoanode in series and a Pt counter electrode, demonstrated a $12.4 \%$ efficiency of conversion of light energy to stored chemical energy in the form of hydrogen and oxygen. ${ }^{7}$ The principal advantage of this tandem or z-scheme configuration (Figure 1b) is that either semiconductor only has to supply part of the photovoltage required to electrolyze water. As a result, materials with smaller bandgaps can be used that absorb more sunlight in the visible and near-infrared region of the solar spectrum. A two-semiconductor system, with both a $p$-type and $n$-type semiconductor photoelectrode, can be configured in several ways. Either the two light absorbers are placed side by side so each one is exposed to the full solar spectrum, or, alternatively, the semiconductors are placed in series, with the larger bandgap semiconductor in front of the smaller bandgap material. In both cases, the two materials must absorb nearly equal numbers of solar photons, since the current through each semiconductor must be matched due to the recombination at the ohmic contacts that provides the summation of the two photovoltages. Because a tandem system requires twice as many photons to split water, the quantum yield is half that of a single photoelectrode system. The advantage of placing semiconductors in series is that one glass substrate, with a transparent conducting layer on both sides, could be used for both materials, reducing the total system costs (see Figure 1b). For the parallel arrangement, two substrates would be required, but they need not be transparent (as in the case of the stacked system discussed previously), so cheaper and more abundant materials may be utilized.

\section{Particulate and colloidal semiconductors}

Shortly after the discovery of photoelectrochemical cells, it was also shown that water could be split into hydrogen and oxygen using self-supported photocatalysts (Figure 2a). These "miniature photoelectrochemical cells" consist of a semiconductor powder or colloid (often a large bandgap metal oxide) to which a noble metal (e.g., Pt) is fused. In one configuration, the catalyst absorbs the light and separates the electron-hole-pair at the metal/semiconductor interface. The electron then reduces water on the noble metal, and the hole oxidizes water on the semiconductor surface. To achieve these functions, the energetics of the photocatalysts must satisfy the same thermodynamic demands as a photoelectrochemical cell. An energy diagram is shown in Figure 3. While most photocatalysts are single bandgap absorbers, some employ the z-scheme configuration, shown on the right side of the diagram. In one of the first systems, Arakawa and co-workers used $\mathrm{Pt}_{-} \mathrm{TiO}_{2}$ (anatase bandgap energy, $E_{\mathrm{g}}=3.2 \mathrm{eV}$ ) for water reduction and $\mathrm{Pt}_{-\mathrm{TiO}_{2}}$ (rutile) for water oxidation. ${ }^{8}$ Since the photocatalyst particles could not be wired up like the photoelectrodes of a photoelectrochemical cell, redox shuttles needed to be employed to ensure directional electron transfer between the two light absorbers.

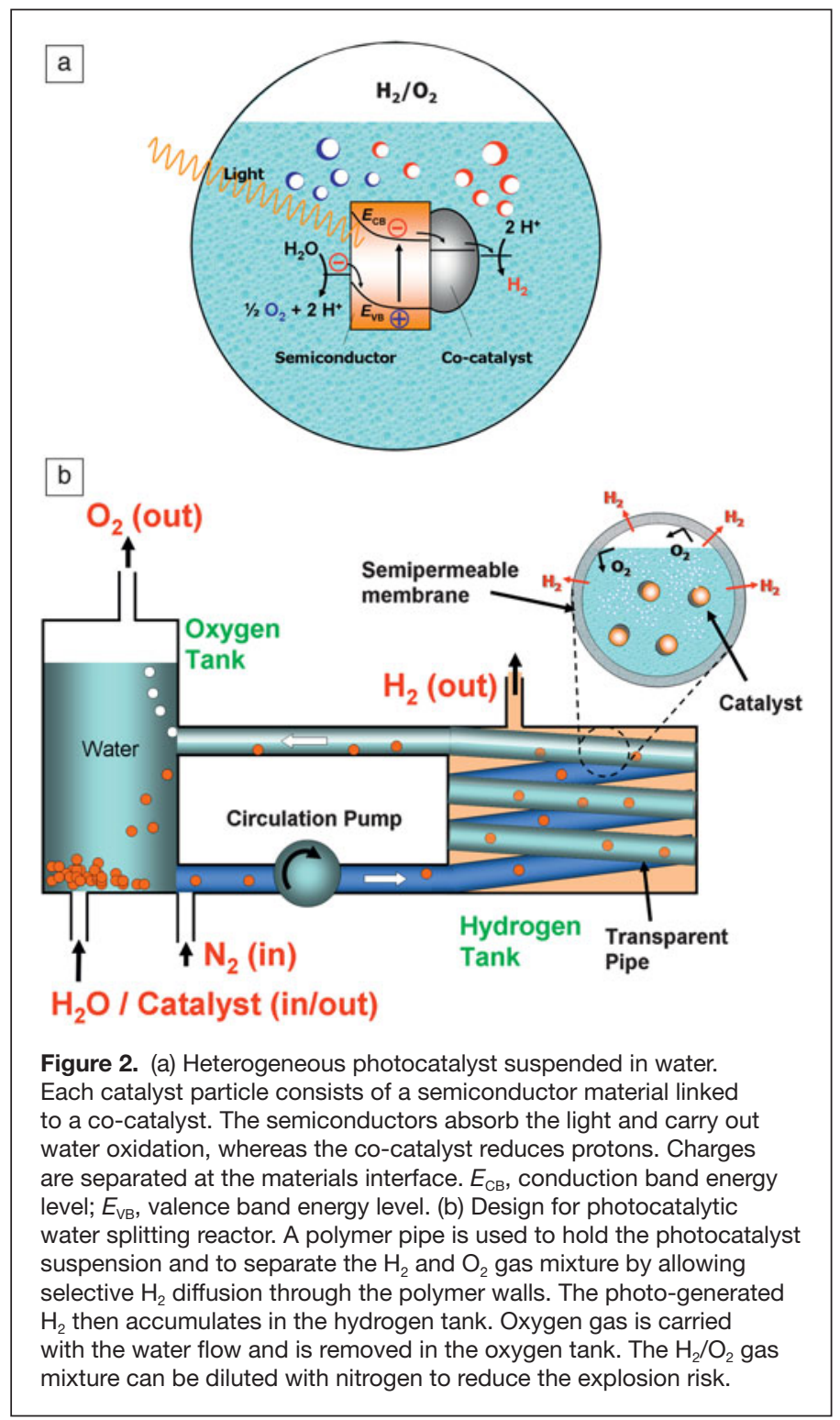

These shuttles must have high specificity for accepting electrons from the photoanodic particles and delivering them to the photocathodic particles.

Compared to photoelectrochemical cells, particulate or colloidal photocatalysts have the advantage that the photolysis can take place in the homogeneous phase without the requirement of expensive transparent electrodes and without the need for directional illumination. On the other hand, one needs to separate the explosive mixtures of hydrogen and oxygen immediately when formed, and this separation process will consume additional energy, reducing the overall efficiency of the water-splitting process. Also, since catalysts for hydrogen and oxygen production from water are often good catalysts for the recombination of hydrogen and oxygen, illumination of such systems without rapid removal of the products will eventually result in a photostationary state, where forward and back reactions have equal rates, and no more net water splitting 
can occur. The photoreactor design in Figure $\mathbf{2 b}$ solves these problems by cycling the catalyst suspension inside a transparent, semipermeable polymer pipe that separates $\mathrm{H}_{2}$ and $\mathrm{O}_{2}$ as they are formed.

For research purposes, when one wants to study only one of the water-splitting half reactions without the complications associated with the kinetics of the other half reaction, sacrificial reagents can be used to scavenge either holes or electrons; however, these additives are not viable for any practical system for sustainable energy production. The most common examples of hole scavenging agents introduced into solution are alcohols (usually methanol), amines (usually triethanolamine or EDTA), or sulfite salts. Electron scavengers, such as the easily reduced $\mathrm{Ag}^{+}$, are also added when studying oxygen evolution.

For suspended photocatalyst powders, metal or metal oxide co-catalysts are often employed to take over the function of the counter electrode in a photoelectrochemical cell (Figure 2a). Co-catalysts can be deposited by soaking the semiconductor powder in a solution of the metal salt followed by reduction with hydrogen gas or photochemically in the presence of a weak electron donor, such as methanol. Under these conditions, co-catalysts often form nanoscale particles. The main purpose of the co-catalysts is to lower the electrochemical overpotentials associated with the multielectron water oxidation and reduction reactions (Figure 3 ). Another function is to provide an interface to enhance electron-hole separation. For water oxidation, the best known catalytic materials are $\mathrm{RuO}_{2}$ and $\mathrm{IrO}_{2}$, whereas for water reduction, noble metals such as $\mathrm{Pt}, \mathrm{Pd}$, and $\mathrm{Ni}$ are often used. As mentioned previously, many co-catalysts (e.g., Pt) catalyze the back reaction of $\mathrm{H}_{2}$ and $\mathrm{O}_{2}$ to water. However, work by Domen and co-workers has shown that a $\mathrm{Cr}_{2} \mathrm{O}_{3}$ nanoscale coating on $\mathrm{Rh}$ particles can inhibit the back reaction by blocking access of $\mathrm{O}_{2}$ to the $\mathrm{Rh}$ particles. ${ }^{9}$ Currently, there is a pressing need for new catalyst materials that do not employ precious metals. The recent report where amorphous Co-oxyphosphates are used as water oxidation catalysts is promising. ${ }^{10} \mathrm{~A}$ different approach involves the synthesis of molecular catalysts made from more abundant and less expensive metals that also offer the flexibility of tuning their properties by rational synthetic control of their structure. The article by Yang et al., in this special issue reports on recent developments in this area.

\section{Maximum theoretical efficiency}

The maximum efficiency of a photovoltaic device is governed by the fraction of the solar energy that can be absorbed and the photovoltage that can be generated, both of which are related to the bandgap or bandgaps of the materials in the device. All conventional devices have thermalization losses from carriers created at energies greater than the bandgap relaxing rapidly to the band edge. These losses constrain the efficiency to the so-called Shockley Queisser efficiency limit for photovoltaic cells, ${ }^{11}$ where the optimum bandgap for a single junction photovoltaic device is around $1.4 \mathrm{eV}$, and the maximum efficiency is about $30 \%$ at 1 sun of illumination. The need to generate a photovoltage of more than $1.23 \mathrm{~V}$, the thermodynamic potential needed to electrolyze water, adds additional considerations for the optimal efficiency for a photoelectrolysis device. The additional voltage that needs to be generated in the device to drive the electrochemical oxidation and reduction of water at current densities that would be generated at one sunlight intensity $\left(15-25 \mathrm{~mA} / \mathrm{cm}^{2}\right)$ adds at least $0.6 \mathrm{~V}$ to the photopotential needed. Therefore, if a single semiconductor absorber is used for water splitting, good catalysis of water oxidation and reduction are achieved using the rule of thumb that a good photovoltaic device generates at most about two-thirds of its bandgap in photovoltage, at 1 sun. Thus, the minimum bandgap needed is greater than $2.0 \mathrm{eV}(0.66 \times 2.1 \mathrm{eV}+0.6 \mathrm{eV})$. This requirement leaves out much of the solar spectrum and reduces the maximum practically achievable efficiency to less than $10 \%$.

When a $p-n$ or tandem photoelectrolysis system is used, the photovoltages in the two semiconductors can add at the expense of the recombination of majority carriers at the contact between the two materials. This allows one to employ lower bandgap materials that can absorb photons, even extending into the near-infrared, where there is considerable solar photon flux. The trade-off is that due to the recombination, 
the quantum yield is reduced by a factor of two, because now two photons are required to generate one electron-hole-pair of sufficient energy to split water. The result is that considerable higher efficiencies can be achieved in these multijunction devices. For a tandem device with two bandgaps of about 1.9 and $1.2 \mathrm{eV}$, a maximum practical water splitting efficiency of about $15 \%$ can be achieved. More detailed treatments of these efficiency limits are given in the literature. ${ }^{12-14}$

\section{Nanostructured materials}

In recent years, nanomaterials have also emerged as efficient components of photoelectrochemical cells and photocatalysts. Because of their small size, photogenerated carriers in nanocrystals are always created near a surface, where water conversion takes place. For example, bulk single-crystal $\alpha-\mathrm{Fe}_{2} \mathrm{O}_{3}$ (hematite) has a very low activity as a photocatalyst because its paramagnetism shortens the lifetimes and diffusion length $(2-20 \mathrm{~nm})$ of its photogenerated carriers, ${ }^{15}$ so they recombine within the material before they reach the electrolyte interface. In addition, the $d-d$ transitions in transition metal oxide semiconductors, which impart visible light absorption in these materials, are forbidden, resulting in low absorption coefficients and carrier creation deep in the material where a space charge field is not present to separate the electron-hole-pairs. Nanocrystalline $\alpha-\mathrm{Fe}_{2} \mathrm{O}_{3}$, on the other hand, can more efficiently photocatalyze water oxidation because the small dimensions of the crystallites (10-20 nm) allow the photogenerated carriers to diffuse to the electrolyte interface and react with water. Furthermore, optimizing the nanostructure of the film can result in absorption of all photons with energies above the bandgap. ${ }^{16}$ In photoelectrolytic cells, such nanoscale effects have led to $50-90 \%$ efficiency gain. ${ }^{15,17,18}$ It is likely that the same principle can be applied to other metal oxides with short carrier diffusion lengths.

For many other semiconductors, quantum size effects can be utilized to increase performance for solar water splitting. By changing the size and shape of semiconductor nanocrystals, the band energies can be modified, and redox potentials of electrons and holes can be tuned. Frame et al. demonstrated for the first time that nano-CdSe $\left(E_{\mathrm{g}}=2.7 \mathrm{eV}\right)$ becomes active for photocatalytic $\mathrm{H}_{2}$ evolution from water containing sulfite/ sulfide as a sacrificial electron donor, while bulk CdSe $\left(E_{\mathrm{g}}=\right.$ $1.7 \mathrm{eV}$ ) is not. ${ }^{19,20}$ The activation is due to a raised conduction band edge $(-0.55 \mathrm{~V}$, normal hydrogen electrode, NHE, $\mathrm{pH}=7$ ) caused by quantum size confinement. As a result, electrons are about $0.45 \mathrm{~V}$ more reducing than those in bulk CdSe $(-0.10 \mathrm{~V}, \mathrm{NHE}, \mathrm{pH}=7)$.

Sambur et al. has recently demonstrated a photovoltaic system with $\mathrm{PbS}$ quantum dots covalently bound to a $\mathrm{TiO}_{2}$ crystal surface that when excited with photons of more than 2.8 times the quantum dot bandgap, can collect two electrons per photon injected into the $\mathrm{TiO}_{2}$ conduction band as photocurrent. ${ }^{31}$ Systems with quantum yields for electron generation of more than one for high-energy photons can reduce some of the losses associated with the Shockley-Queisser limit, as discussed previously, but this multiple exciton collection has not yet been demonstrated in a water-splitting system.

\section{Combinatorial techniques for discovery and optimization of photocatalysts}

It is primarily for their stability that metal oxide semiconductors arguably hold the most promise for constructing a stable photoelectrolysis system. However, many of the early transition metal oxides are wide bandgap materials that do not absorb a sufficient amount of the solar spectrum. Several research directions have emerged to overcome this limitation. They include nitrogen incorporation into the metal oxide lattice and combinatorial metal oxide syntheses. While metal oxynitrides will be discussed in the article by Maeda and Domen, combinatorial techniques will be briefly discussed here.

Multicomponent materials are often required when a material with special properties needs to be discovered or optimized. Elements specific to performing each of the required roles in the overall functionality of these special materials need to be combined in specific proportions in order to create the desired synergy. High-temperature superconductivity is a good example of a special property requiring multicomponent materials. The superconducting oxides with the highest known transition temperatures contain four or five metals $\left(\mathrm{HgBa}_{2} \mathrm{CaCu}_{2} \mathrm{O}_{6+\delta}\right.$ and $\mathrm{Hg}_{0.8} \mathrm{Tl}_{0.2} \mathrm{Ba}_{2} \mathrm{Ca}_{2} \mathrm{Cu}_{3} \mathrm{O}_{8.33}$ ). Since our present theoretical knowledge is insufficient to a priori calculate the behavior of such complex systems or estimate the identity and atomic ratios of the specific metals needed to perform the efficient photoelectrolysis of water, for now empirical methods will be necessary to identify the best material. Hundreds of thousands or perhaps millions of mixed metal oxide photocatalyst compositions may need to be produced and tested for efficiency and corrosion resistance until an effective material is discovered.

Combinatorial methods provide tools to speed the discovery process when a large number of candidate materials need to be synthesized and screened for the property of interest. ${ }^{21}$ Baeck and MacFarland have demonstrated the electrochemical deposition of metal oxide compositions using robotics to plate and screen individually created materials and have looked at water photo-oxidation catalysts using multiplexed photoelectrochemical cells. ${ }^{22}$ Woodhouse and Parkinson have developed a high throughput combinatorial search strategy for the identification of multicomponent metal oxide materials with suitable bandgaps and band positions for water photoelectrolysis operating as either a photoanode or a photocathode. Their combinatorial search strategy uses ink jet printing of overlapping patterns of metal oxide precursors onto conductive glass substrates. ${ }^{23}$ The simplest metal oxide precursors are metal nitrates that decompose to form metal oxides, oxygen, and $\mathrm{NO}_{2}$ by heating at relatively low temperatures $\left(<500^{\circ} \mathrm{C}\right)$. The low cost, speed, and versatility of ink jet printing have already made it attractive for combinatorial searches for fuel cell catalysts, ${ }^{21,24}$ organic device combinations, ${ }^{25}$ and biomaterials. ${ }^{26}$ We feel that the ink jetting approach has several major advantages for identifying oxide photoelectrolysis materials. It is a simple method, and 


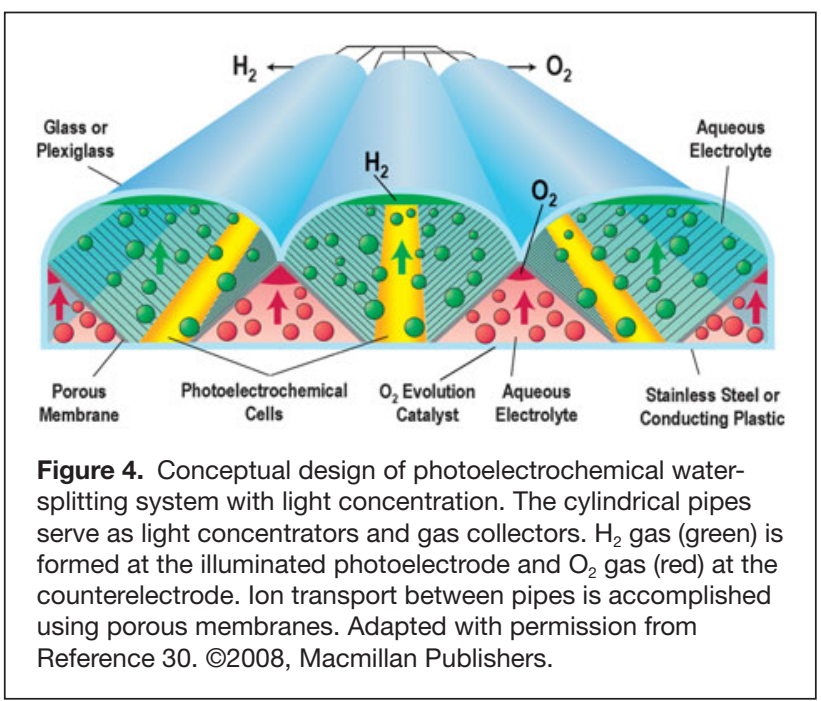

even inexpensive off-the-shelf ink jet printers can be used. It can be adapted to broad screening of compositions for discovery or very fine control of stoichiometry and doping for optimization. We have used this approach to discover several new promising oxide semiconductors. ${ }^{27}$ Since there are so many possible combinations to produce and screen, we have developed a project to enlist a multitude of undergraduates and high school students to help with the effort to discover these most important materials. The Solar Hydrogen Activity research Kit, or SHArK Project, uses simple materials such as Lego Mindstorms to provide the students with everything they need to produce libraries of metal oxides and screen them for photoelectrolysis activity. ${ }^{28,29}$

\section{Additional design criteria}

Besides high efficiency, there are additional criteria that need to be considered for a successful solar water-splitting system. As stated previously, the photoactive materials must be inexpensive and very stable against photocorrosion to allow an energy and financial return from the initial investment. Any photoelectrochemical cell will have to be designed with an ion-conducting separator or electrolyte path between the two electrodes and with separate gas collection plumbing for hydrogen and perhaps oxygen (a conceptual photoelectrochemical device is shown in Figure 4). These supporting structures, electrolyte and gas handling systems, must also last for many years. Since almost none of these design criteria can be met with current materials, it is clear that there is a strong need for basic research in solar water splitting and for many researchers to get involved in the discovery and optimization of these materials.

\section{Acknowledgments}

Frank Osterloh thanks the National Science Foundation (NSF Grant 0829142) for support and Research Corporation for Science Advancement for a Scialog award. Bruce Parkinson acknowledges the U.S. Department of Energy, Office of Science, NSF, and the University of Wyoming for support of his research in photoelectrochemical energy conversion.

\section{References}

1. N.S. Lewis, D.G. Nocera, Proc. Nat. Acad. Sci. U.S.A. 103, 15729 (2006)

2. T. Kodama, N. Gokon, Chem. Rev. 107, 4048 (2007).

3. S. Abanades, P. Charvin, G. Flamant, P. Neveu, Energy 31, 2805 (2006).

4. P.J. Boddy, J. Electrochem. Soc. 115, 199 (1968).

5. A. Fujishima, K. Honda, Nature 238, 37 (1972).

6. A. Fujishima, K. Honda, Bull. Chem. Soc. Jpn. 44, 1148 (1971).

7. 0. Khaselev, J.A. Turner, Science 280, 425 (1998).

8. R. Abe, K. Sayama, K. Domen, H. Arakawa, Chem. Phys. Lett. 344, 339 (2001)

9. K. Maeda, K. Teramura, D.L. Lu, N. Saito, Y. Inoue, K. Domen, Angew. Chem. Int. Ed. 45, 7806 (2006).

10. Y. Surendranath, M. Dincă, D.G. Nocera, J. Am. Chem. Soc. 131, 2615 (2009).

11. W. Shockley, H.J. Queisser, J. Appl. Phys. 32, 510 (1961).

12. J.R. Bolton, S.J. Strickler, J.S. Connolly, Nature 316, 495 (1985).

13. O.K. Varghese, C.A. Grimes, Sol. Energy Mater. Sol. Cells 92, 374 (2008).

14. M.F. Weber, M.J. Dignam, Int. J. Hydrogen Energy 11, 225 (1986).

15. I. Cesar, A. Kay, J.A.G. Martinez, M. Gratzel, J. Am. Chem. Soc., 128, 4582 (2006).

16. I. Cesar, K. Sivula, A. Kay, R. Zboril, M. Grätzel, J. Phys. Chem. C 113, 772 (2009).

17. A. Duret, M. Grätzel, J. Phys. Chem. B 109, 17184 (2005).

18. D.K. Zhong, J.W. Sun, H. Inumaru, D.R. Gamelin, J. Am. Chem. Soc. 131 6086 (2009)

19. F.A. Frame, E.C. Carroll, D.S. Larsen, M.S. Sarahan, N.D. Browning, F.E. Osterloh, Chem. Commun. 2206 (2008).

20. F.A. Frame, F.E. Osterloh, J. Phys. Chem. C, 114, 10628 (2010).

21. E. Reddington, A. Sapienza, B. Gurau, R. Viswanathan, S. Sarangapeni, E.S. Smotkin, T.E. Mallouk, Science 280, 1735 (1998).

22. S.H. Baeck, T.F. Jaramillo, C. Brandli, E.W. McFarland, J. Comb. Chem. 4 563 (2002).

23. M. Woodhouse, G.S. Herman, B.A. Parkinson, Chem. Mater. 17, 4318 (2005). 24. P. Cong, R.D. Doolen, Q. Fan, D.M. Giaquinta, S. Guan, E.W. McFarland, D. Poojary, K. Self, H.W. Turner, W.H. Weinberg, Angew. Chem. Int. Ed. 38, 484 (1999).

25. H. Sirringhaus, T. Kawase, R.H. Friend, T. Shimoda, M. Inbasekaran, W. Wu, E.P. Woo, Science 290, 2123 (2000).

26. G. MacBeath, S.L. Schreiber, Science 289, 1760 (2000).

27. M. Woodhouse, B.A. Parkinson, Chem. Mater. 20, 2495 (2008).

28. B. Parkinson, Energy Environ. Sci. 3, 509 (2010).

29. www.thesharkproject.org.

30. J. Turner, Nat. Mater. 7, 770 (2008).

31. J.B. Sambur, T. Novet, B.A. Parkinson, Science, 330, 63 (2010).

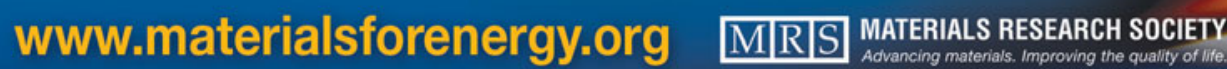

\section{Join the Conversation in the Materials for Energy Blog}

- READ about bioremediation for oil spills, materials availability for electric vehicles, or the hottest superconductors

- CONTRIBUTE your thoughts to the developing stories

- SUBSCRIBE to the Materials for Energy RSS feed

- SHARE the news with friends and colleagues-Link to Facebook, LinkedIn, Twitter and other social media 